\title{
Diagnostische Urteile von Lehrkräften erklären - Ein Rahmenmodell für kognitive Modellierungen und deren experimentelle Prüfung
}

\author{
Timo Leuders · Katharina Loibl · Tobias Dörfler
}

Eingegangen: 24. September 2020 / Überarbeitet: 24. September 2020 / Angenommen: 25. September 2020 / Online publiziert: 8. Oktober 2020

(C) Der/die Autor(en) 2020

Zusammenfassung Der Forschungsstand zu diagnostischen Kompetenzen von Lehrkräften - als wesentliche Voraussetzung für Feedback oder adaptives Unterrichten - wird zurzeit als unbefriedigend angesehen, da kaum überzeugende Theorien über die kognitiven Prozesse bei der Genese diagnostischer Urteile bestehen. An dieser Stelle setzt das Rahmenmodell DiaCoM (Explaining Teachers' Diagnostic Judgements by Cognitive Modeling) an. Das DiaCoM-Rahmenmodell bietet eine theoretische Basis für Forschungsansätze, die diagnostische Urteile von Lehrkräften als Informationsverarbeitungsprozesse erklären wollen. Es konzeptualisiert diagnostisches Urteilen in Bildungskontexten als kognitive Prozesse einer Lehrkraft über Schülerinnen und Schüler (z. B. deren Fähigkeit) oder über Anforderungen (z. B. Aufgabenschwierigkeiten) auf der Grundlage der Informationen, die explizit oder implizit in einer diagnostischen Situation bestehen. Es bezieht sich auf Theorien der kognitiven Informationsverarbeitung und erfordert eine Spezifikation von vier Komponenten: die Personencharakteristika, die Situationscharakteristika, das diagnostische Denken als Informationsverarbeitung und schließlich das Diagnoseverhalten. Der Beitrag stellt dar, wie das DiaCoM-Rahmenmodell als forschungsheuristisches Modell eingesetzt werden kann, um Erklärungswissen zur Genese diagnostischer Urteile zu generieren: Durch Spezifikation der informationsverarbeitenden Prozesse können theoretische Voraussagen darüber getroffen werden, welche Personen- und Situationscharakteristika zu welchem diagnostischen Verhalten führen. Diese Annahmen sind dann einer experimentellen Prüfung durch systematische Variation der Situation oder der Personen (z. B. durch Instruktion) zugänglich.

\footnotetext{
T. Leuders $(\varangle) \cdot$ K. Loibl

Pädagogische Hochschule Freiburg, Freiburg, Deutschland

E-Mail: leuders@ph-freiburg.de

T. Dörfler

Pädagogische Hochschule Heidelberg, Heidelberg, Deutschland
} 
Schlüsselwörter Diagnostische Kompetenz · Diagnostische Urteile · Lehrkräfte · Informationsverarbeitung · Forschungsheuristisches Rahmenmodell

\title{
Explaining diagnostic judgments of teachers-a framework for cognitive models and their experimental testing
}

\begin{abstract}
The state of research on diagnostic skills of teachers-as an essential prerequisite for feedback or adaptive teaching-is currently considered unsatisfactory, as there are hardly any convincing theories on the cognitive processes involved in the genesis of diagnostic judgements. This is where the DiaCoM framework (Explaining Teachers' Diagnostic Judgements by Cognitive Modeling) comes in. The DiaCoM framework provides a theoretical basis for research approaches that aim to explain diagnostic judgements of teachers as information processing. It conceptualizes diagnostic judgments in educational contexts as cognitive processes of a teacher about students (e.g., their ability) or about demands (e.g., task difficulties) based on the information that is explicitly or implicitly present in a diagnostic situation. It refers to theories of cognitive information processing and requires a specification of four components: personal characteristics, situation characteristics, diagnostic thinking as information processing, and finally diagnostic behavior. The paper shows how the DiaCoM framework can be used as a heuristic model for research to generate explanatory knowledge about the genesis of diagnostic judgements: By specifying the information processing, theoretical predictions can be made about which person and situation characteristics lead to which diagnostic behavior. These assumptions can then be tested experimentally by systematic variation of the situation or persons (e.g. by instruction).
\end{abstract}

Keywords Diagnostic competence - Diagnostic judgements · Teachers · Information processing $\cdot$ Cognitive modeling $\cdot$ Heuristic model for research

\section{Einleitung}

Diagnostizieren gilt als eine wesentliche Tätigkeit von Lehrkräften: Eine zutreffende Einschätzung über Wissen, Motivation oder Denken der Schülerinnen und Schüler ist Voraussetzung für das Feedback an Lernende oder Eltern, für Übergangsentscheidungen im Bildungssystem, für die Anpassung des Unterrichts (z. B. nach Fähigkeitsniveau), aber auch für die Einteilung eigener Ressourcen (z.B. Artelt und Gräsel 2009; Baumert und Kunter 2013; Glock et al. 2015; Helmke 2010; Hobfoll et al. 2018; Praetorius et al. 2013). Das Wissen und die Fähigkeiten der Lehrkräfte, die für die Vorbereitung, Durchführung und Reflexion von Beurteilungen erforderlich sind, werden unter dem Oberbegriff diagnostische Kompetenz zusammengefasst (Herppich et al. 2018; Leuders et al. 2018; Popham 2009; Schrader 2009; Weinert et al. 1990). Wenn eine Lehrkraft einen Schüler bzw. eine Schülerin, eine Aufgabe oder allgemeiner eine Lehr-Lern-Situation beurteilt, werden sowohl der Prozess als auch das Ergebnis dieser Diagnose allgemein als diagnostisches Urteil bezeichnet (Artelt und Rausch 2014). Die Qualität eines diagnostischen Urteils wird traditionell 
anhand seiner Akkuratheit bewertet (Anders et al. 2010; Hoge und Coladarci 1989; Spinath 2005; Südkamp et al. 2012; Schrader 1997). Dabei interessieren z. B. folgende Fragen: Wie gut stimmt ein Urteil der Lehrkraft mit einer objektiven Bewertung der Merkmale des Schülers oder der Schülerin (z. B. Fähigkeit) oder des Materials (z.B. Aufgabenschwierigkeit) überein? Jede systematische Abweichung dieser Genauigkeit wird als Beurteilungsfehler betrachtet (wie in allgemeinen Theorien über soziale Urteile; z. B. Kahneman 2003), den es zu vermeiden gilt.

Studien zur diagnostischen Kompetenz von Lehrkräften konzentrieren sich auf verschiedene Aspekte. Die meisten Forschungsarbeiten zielen darauf ab, den Einfluss verschiedener situativer Faktoren (z. B. der untersuchten Lerngruppe oder der Aufgabenschwierigkeiten) oder der Eigenschaften von Lehrpersonen (z. B. Berufserfahrung oder Fachwissen) auf die Urteilsgenauigkeit zu analysieren (für einen Überblick siehe Südkamp et al. 2012). Andere Ansätze sind an der Untersuchung des breiten Spektrums von Lehrkräfteaktivitäten im Zusammenhang mit Diagnosen interessiert (z. B. Black und Wiliam 2003; Herppich et al. 2018). Der Forschungsstand $\mathrm{zu}$ diagnostischem Urteilen wird in der Bildungsforschung jedoch als unbefriedigend angesehen, da bisher noch keine überzeugenden Theorien über die kognitiven Prozesse beim Diagnostizieren aufgestellt wurden (Schrader 2009; Spinath 2005; Leuders et al. 2018; Herppich et al. 2018). Für das Verständnis der Genese eines Urteils ist es jedoch entscheidend, welche Informationen in der Diagnosesituation von einer Lehrkraft wahrgenommen werden und wie diese Informationen verarbeitet werden. Überraschenderweise ist bislang nur wenig über diese Informationsverarbeitungsprozesse von Lehrkräften bekannt.

An dieser Stelle setzt das Rahmenmodell DiaCoM (Explaining Teachers' Diagnostic Judgements by Cognitive Modeling; Erklärung der diagnostischen Urteile von Lehrkräften durch kognitive Modellierung; Loibl et al. 2020) an. Das DiaCoM-Rahmenmodell bietet eine theoretische Basis für Forschungsansätze, die diagnostische Urteile von Lehrkräften als Informationsverarbeitungsprozesse erklären wollen. Die Beiträge in diesem Heft nehmen expliziten Bezug zum DiaCoM-

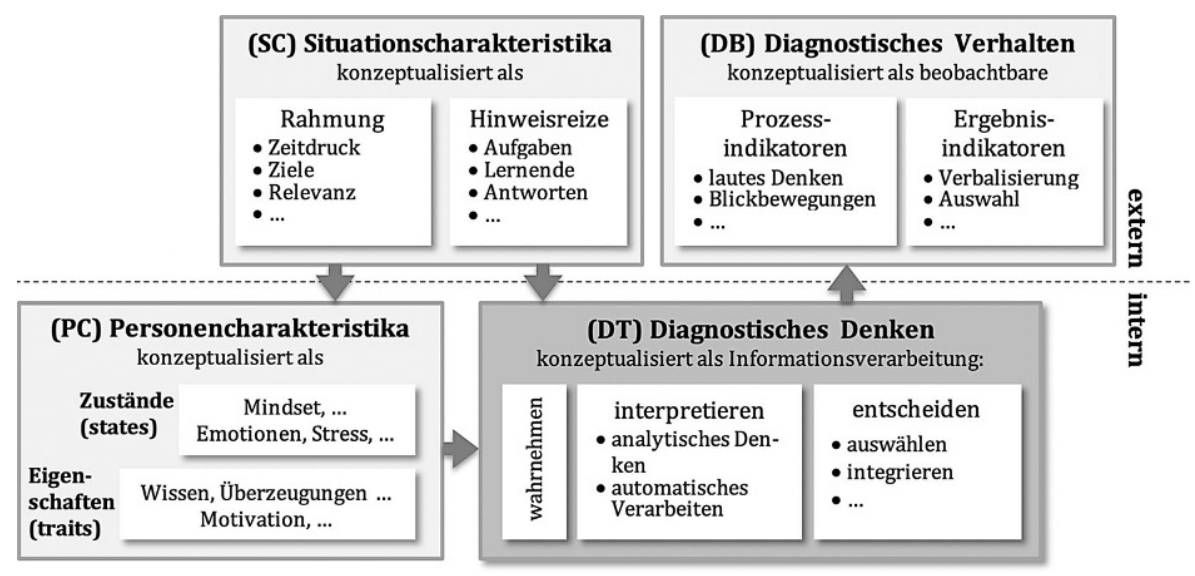

Abb. 1 Komponenten des DiaCoM-Rahmenmodells. (Loibl et al. 2020) 
Rahmenmodell und haben das Ziel, Erklärungswissen zum Entstehen diagnostischer Urteile zu generieren. Das Rahmenmodell wird im Folgenden in seiner Struktur und seiner forschungsstrategischen Funktion vorgestellt. Der Text orientiert sich dabei an der ausführlichen Darstellung bei Loibl et al. (2020).

\section{Das DiaCoM-Rahmenmodell und seine Komponenten}

Das DiaCoM-Rahmenmodell (Loibl et al. 2020) konzeptualisiert diagnostisches Urteilen in Bildungskontexten als kognitive Prozesse einer Lehrkraft über Schülerinnen und Schüler (z. B. deren Fähigkeit) oder über Anforderungen (z. B. Aufgabenschwierigkeiten) auf der Grundlage der Informationen, die explizit oder implizit in einer diagnostischen Situation bestehen. Es bezieht sich auf Theorien der kognitiven Informationsverarbeitung und beschreibt diese zunächst auf generische Weise. Dabei werden im DiaCoM-Rahmenmodell nicht alle für eine diagnostische Unterrichtssituation relevanten Aspekte abgebildet: Zum einen haben Lehrkräfte im DiaCoMRahmenmodell eine definierte Rolle als urteilende Subjekte und Schülerinnen und Schüler als Objekte von Urteilen. Das heißt, dass z. B. Prozesse der Selbsteinschätzung von Schülerinnen und Schülern oder die Metakognition von Lehrkräften über ihr eigenes Urteil hier nicht einbezogen werden. Zum zweiten werden komplexe Handlungen von Lehrkräften im Umfeld der diagnostischen Urteile, wie z. B. das Generieren von Diagnoseaufgaben oder das Führen diagnostischer Gespräche, im DiaCoM-Rahmenmodell nicht berücksichtigt, da wir uns auf kognitive Informationsverarbeitungsprozesse konzentrieren, die an der Entstehung diagnostischer Beurteilungen beteiligt sind. Demgegenüber beziehen Ansätze anderer Forschungsgruppen (cosima: Heitzmann et al. 2019; NeDiKo: Herppich et al. 2018) solche Aktivitäten von Lehrkräften in diagnostischen Situationen mit ein.

Innerhalb dieses Fokus spezifiziert das DiaCoM-Rahmenmodell vier Komponenten, die in Abb. 1 dargestellt und in den folgenden Abschnitten erläutert werden: die Personencharakteristika (d. h. die Merkmale der Lehrkraft) (PC), die Situationscharakteristika (SC), das diagnostische Denken (DD) als Informationsverarbeitung und schließlich das Diagnoseverhalten (DV). Dabei soll das DiaCoM-Rahmenmodell nicht eo ipso als kognitives Modell diagnostischen Denkens verstanden werden, sondern vielmehr als ,forschungsheuristisches Modell“. Zentrale Funktion des DiaCoM-Rahmenmodells ist es Forschungsprozesse zu strukturieren, welche das Ziel haben, Erklärungswissen zur Genese diagnostischer Urteile zu generieren. Dies wird dadurch möglich, dass nach Spezifikation der informationsverarbeitenden Prozesse theoretische Voraussagen darüber getroffen werden können, welche Personen- und Situationscharakteristika zu welchem diagnostischen Verhalten führen. Solche Zusammenhänge sind dann einer experimentellen Prüfung durch systematische Variation der Situation oder der Personen (z. B. durch Instruktion) zugänglich.

Die Komponente des diagnostischen Denkens (DD) ist das Herzstück des DiaCoM-Rahmenmodells. Diagnostisches Denken wird im Modell als kognitive Informationsverarbeitung konzeptualisiert. Informationen stammen aus der Situation oder werden im Langzeitgedächtnis gespeichert. Somit kann die Informationsverarbeitung durch stabile Merkmale (z. B. Überzeugungen) oder vorübergehende Zustände 
(z. B. Stress) der Lehrperson moderiert (gefiltert, geändert, modifiziert usw.) werden. Obwohl das DiaCoM-Rahmenmodell die kognitive Modellierung des diagnostischen Denkens hervorhebt, schlagen wir kein allgemeingültiges kognitives Modell für alle diagnostischen Situationen oder Forschungsfragen vor. Stattdessen muss diese Komponente des Rahmenmodells in Bezug auf das jeweilige Forschungsziel spezifiziert werden, indem auf der breiten Literatur zu Informationsverarbeitungsmodellen aufgebaut wird - z. B. zum Urteilen und Entscheiden (Gigerenzer und Gaissmaier 2011), zum Problemlösen (Newell und Simon 1972; Schoenfeld 1985) oder zum induktiven Schließen (z. B. Feeney und Heit 2007). Die möglichen Spezifikationen sind so vielfältig wie die diagnostischen Urteilsprozesse, die beschrieben werden sollen. Konkrete Beispiele finden sich in den in diesem Heft vorgestellten Studien sowie in den Beispielstudien in Loibl et al. (2020).

Die Personencharakteristika (PC) umfassen alle Merkmale der Lehrperson (z. B. Wissen, Überzeugungen, Motivation), die die Beurteilung eines schulischen Diagnosegegenstandes beeinflussen können. Wissen kann allgemein (z. B. Wissen über pädagogische Inhalte über Schülertypen) oder spezifisch in Bezug auf Schülerinnen und Schülern oder Aufgaben sein (z. B. vermutete Eigenschaften der Schülerinnen und Schüler, Kenntnis der Lösungen der Schülerinnen und Schüler oder typische Fehler bei bestimmten Aufgaben). Diese Konzeptualisierung von Eigenschaften (Traits) ermöglicht die Formulierung theoretischer Annahmen über die Verwendung von Wissen und/oder den Einfluss von wissensbasierten Überzeugungen während des Urteilsprozesses. Um solche theoretischen Annahmen zu testen, ist es notwendig, die Eigenschaften von Lehrkräften $\mathrm{zu}$ messen oder experimentell zu manipulieren (z. B. durch gezielte Instruktionen vor der Beurteilung einer diagnostischen Situation). Bestimmte Situationen können zudem einen temporären kognitiven, motivierenden oder emotionalen Zustand (State, z. B. Stress) auslösen, der den Beurteilungsprozess beeinflussen kann. Abhängig von der Forschungsfrage kann es daher wichtig sein, den Zustand der Lehrkraft als situationsspezifisches Merkmal der Person zu manipulieren und/oder zu messen. Die meisten Lehrkräfteeigenschaften (wie motivationale Dispositionen oder intellektuelle Fähigkeiten) sind schwer zu trainieren. Wissensfacetten sowie ausgewählte situative Merkmale stellen stattdessen Komponenten im Modell dar, die durch Interventionen leicht verändert werden können.

Innerhalb des DiaCoM-Rahmenmodells geben die Situationscharakteristika (SC) den Kontext vor, auf den die Lehrkraft stößt oder der von der Lehrkraft bewusst oder unbewusst erzeugt wird. Diese diagnostische Situation enthält die Informationen, die die Lehrperson nutzen kann, um Rückschlüsse auf Materialien oder Schülerinnen und Schüler zu ziehen. Im DiaCoM-Rahmenmodell müssen diese Informationen explizit durch Cues definiert werden (vgl. Brunswik 1955). Cues stellen diejenigen Informationen dar, die beim Diagnostizieren verwendet werden können. Einige Cues können leicht wahrgenommen werden (sog. Oberflächenmerkmale, d. h. Merkmale, die durch niedrig-inferenzielle Informationsverarbeitung identifiziert werden können), während Tiefenstrukturmerkmale nur mit hohem kognitiven Aufwand und durch hoch-inferenzielle oder komplexe Informationsverarbeitung erkannt und eingeordnet werden können. Ob eine Situation tatsächlich einen bestimmten Cue enthält, kann theoretisch festgelegt werden - etwa bei systematischer Konstruktion ei- 
ner Situation - oder durch die Befragung von Expertinnen und Experten empirisch bestimmt werden. Cues können sich auf Aufgaben, auf Schülerinnen und Schüler oder auf Aspekte des Kontextes (z. B. die Klassenkomposition) beziehen. Bei der Spezifizierung des DiaCoM-Rahmenmodells bedarf es einer bewussten und auf den Forschungszielen basierenden Entscheidung zur Auswahl und Konzeptualisierung der Cues. Durch Auswahl geeigneter Cues kann eine valide theoretische Darstellung der Situation erreicht werden. Durch systematisch variierende Cues können experimentell kontrollierte Bedingungen erzeugt und Theorien über die Verwendung dieser Cues während der Informationsverarbeitung validiert werden. Experimente zur CueNutzung sind vielversprechend, da die Informationsdichte oder das Auftreten von Cues in der Forschung leicht manipuliert werden können.

Schließlich bezieht sich die Komponente des diagnostischen Verhaltens (DV) auf alle Arten von beobachtbarem Verhalten von Lehrerkräften. Das diagnostische Verhalten kann sowohl im Hinblick auf den Beurteilungsprozess als auch auf das Beurteilungsergebnis betrachtet werden. Bevor das diagnostische Urteil gefällt wird, können Augenbewegungen, Protokolle zum Lauten Denken oder andere Prozessdaten verwendet werden, um auf die zugrundeliegenden Beurteilungsprozesse (das diagnostische Denken) zu schließen. Mündliche oder schriftliche Antworten sowie (Unterrichts-)Handlungen können als Indikator für das Beurteilungsergebnis herangezogen werden. Dabei ist der Übergang zwischen diagnostischem Verhalten im Prozess und als Ergebnis fließend.

Die Anwendung des DiaCoM-Rahmenmodells beschränkt sich auf die Prozesse und Ergebnisse, die mit der sofortigen Beurteilung nach der Darstellung einer diagnostischen Situation verbunden sind. Aktivitäten der Lehrkraft wie das Beschaffen weiterer Informationen (über die Klasse oder aus der Fachliteratur) oder die Entscheidung über eine Unterrichtsstrategie werden absichtlich nicht im DiaCoM Rahmenmodell betrachtet, um eine kontrollierte Erforschung der fokussierten Prozesse zu ermöglichen. Andere Modelle umfassen solche weiterführenden Prozesse (NeDiKo: Herppich et al. 2018 und cosima: Heitzmann et al. 2019). Obwohl das DiaCoMRahmenmodell so nur einige Aspekte der diagnostischen Aktivitäten von Lehrkräften abdeckt, kann es immer noch erhebliche Unterschiede bei den diagnostischen Urteilen hinsichtlich der Struktur der Diagnosesituation, der Einflussfaktoren und der potenziell relevanten kognitiven Prozesse erklären.

Zusammenfassend konzentrierte sich bisher der überwiegende Teil der Forschung zu diagnostischen Urteilen von Lehrkräften auf die Genauigkeit der Urteile im Vergleich zu (standardisierten) Tests. Für diesen Forschungsansatz wurden bereits einige relevante Faktoren identifiziert, die korrelativ mit der Urteilsgenauigkeit in Beziehung stehen (vgl. Südkamp et al. 2012). Korrelationsstudien können die Genese der Urteile durch kognitive Prozesse jedoch nicht erklären. Mit dem DiaCoM-Rahmenmodell schlagen wir eine Grundlage vor, um Hypothesen und Studiendesigns abzuleiten, die sich auf kognitive Modellierungen konzentrieren und so dazu beitragen, den Prozess des diagnostischen Denkens in Bildungskontexten zu verstehen. Genau diese Strategie wird in den Beiträgen des Themenhefts verfolgt. 


\section{Das DiaCoM-Rahmenmodell als forschungsstrategischer Rahmen}

Die Genese diagnostischer Urteile ist das Ergebnis unterschiedlicher kognitiver Prozesse. Die Nutzung und das Ergebnis der kognitiven Informationsverarbeitung werden vom Typ der Diagnosesituation und vom Diagnosegegenstand beeinflusst. Wie oben ausgeführt, wurden erst in wenigen Bereichen solche kognitiven Prozesse der Urteilsgenese näher untersucht. Die Beiträge dieses Themenhefts setzen an diesem Desiderat an. In allen Beiträgen werden theoretisch fundierte Modelle der Genese diagnostischer Urteile, verstanden als Informationsverarbeitungsprozesse, in spezifischen Diagnosesituationen experimentell auf ihre Geltung geprüft und so Erklärungswissen zu diagnostischen Urteilsprozessen generiert.

1. In jedem Beitrag ist ein konkreter Typ einer diagnostischen Situation spezifiziert (z. B. Einschätzung von Lösungen, Aufgaben, mit/ohne Zeitdruck usw.).

2. Jeder Beitrag fokussiert auf einen konkreten Diagnosegegenstand. Dieser ist einerseits in einem konkreten Schulfach curricular zentral und andererseits in der Forschung bereits so intensiv behandelt, dass es dazu konsolidierte Erkenntnisse auf der Schülerebene gibt (z. B. Schwierigkeiten in Aufgaben, Fehlvorstellungen von Lernenden usw.).

3. In diesem Rahmen werden in jedem Beitrag diagnostische Urteile als Informationsverarbeitungsprozesse theoretisch beschrieben und das hier beschriebene Rahmenmodell damit ausdifferenziert: Prozesse der Wahrnehmung von Hinweisreizen (Cues) in der Situation, Prozesse der Interpretation im Sinne einer wissensgesteuerten Kategorisierung, Prozesse der Entscheidung z. B. als Gewichtung unterschiedlicher Informationen. Die Annahmen über solche Prozesse variieren in den Beiträgen. Die Forschungsfragen und das Design der Projekte sind schließlich auf eine möglichst experimentell angelegte empirische Prüfung der jeweiligen Annahmen über diagnostische Urteilsprozesse ausgerichtet: Die Situation kann systematisch über Hinweisreize variiert werden, die Personenvariablen werden durch Interventionen kontrolliert verändert, die Einflüsse als Vorhersagen auf diagnostisches Verhalten operationalisiert und sodann experimentell überprüft.

Die hier angedeutete Strategie zur Generierung von Erklärungswissen zu diagnostischen Urteilsprozessen wird in Loibl et al. (2020) ausführlicher dargestellt und auf bestehende Studien zu diagnostischen Urteilen angewendet. Im Folgenden wird der forschungsstrategische Nutzen des Modells anhand der Beiträge des vorliegenden Themenhefts illustriert.

Der Beitrag von Rieu, Loibl, Leuders und Herppich fokussiert auf die Genese diagnostischer Urteile über Mathematikaufgaben aus der Bruchrechnung (DV), konkret die relative Schwierigkeit von Aufgaben im paarweisen Vergleich, die sich systematisch bezüglich ihrer schwierigkeitsgenerierenden Merkmale (SC) unterscheiden. Es wird angenommen, dass solche Urteile entstehen, indem schwierigkeitsrelevante Aufgabenmerkmale in Aufgaben identifiziert und gewichtet werden (DD). Die Manipulation des hierzu nötigen fachdidaktischen Wissens (PC), und die systematische Variation der präsentieren Merkmale (cues) und der verfügbaren Zeit (SC) erlauben eine experimentelle Prüfung dieser Annahme über die Informationsverarbeitungs- 
prozesse: angehende Lehrkräfte verknüpfen bei der Aufgabendiagnose tatsächlich systematisch die Aufgabenmerkmale und ihr spezifisches fachdidaktisches Wissen.

Auch im Beitrag von Becker, Spinath, Ditzen und Dörfler werden diagnostische Urteile über die Schwierigkeit von Mathematikaufgaben (DV) in den Blick genommen. Die Autorinnen und der Autor stellen in ihrer Studie die Annahme ins Zentrum, dass unter psychischer Anspannung wie Stress (State, PC) kognitive Kapazitäten gebunden werden, was informationsverarbeitende Prozesse (DD) beim Diagnostizieren von Aufgabenschwierigkeiten, beeinträchtigen kann. Mit einer Kombination von Eye-Trackingdaten, Verbalprotokollen und Cortisolwerten aus Speichelproben sowie einer experimentellen Stressinduktion können sie ihre Erklärung diagnostischer Urteile empirisch untermauern.

Witzigmann und Sachse untersuchen in ihrem Beitrag die Urteile von Französischlehrkräften zu mündlichen Sprachproduktionen von Kindern (DV). Sie explorieren dabei, in welchem Umfang fachdidaktische Expertinnen und Experten dabei auf eine Vielzahl von Merkmalen auf inhaltlicher und sprachlicher Ebene (z.B. hinsichtlich Aussprache, Wortschatz aber auch pragmatischen Elementen) zurückgreifen (DD). Der Urteilsprozess wird dabei durch holistische Urteile einerseits und ein analytisches Raster andererseits strukturiert. Die Befunde können als Ausgangspunkt für systematischere Untersuchungen der bislang noch nicht erforschten Genese von Urteilen über mündliche Sprachproduktionen dienen.

Hoppe, Renkl und Rieß untersuchen, wie (angehende) Lehrkräfte die Vorstellungen von Schülerinnen und Schülern zu ökologischen Konzepten diagnostizieren (DV). Das hierzu nötige (fachdidaktische) diagnostische Wissen (PC) wurde erst im Zusammenhang mit einem Training wirksam und auch nur bei nicht zu komplexem Diagnosegegenstand. Die relative Flüchtigkeit der Information in Videos im Vergleich zu Textvignetten (SC) beeinflusste die Prozesse der Informationsverarbeitung (DD) jedoch nicht negativ.

Förderung Die Studien zu diagnostischen Urteilen von Lehrkräften entstanden im Rahmen eines vom Ministerium für Wissenschaft, Forschung und Kunst des Landes Baden-Württemberg geförderten Forschungs- und Nachwuchskollegs „Diagnostische Kompetenzen von Lehrkräften (DiaKom): Einflüsse, Struktur und Förderung“.

Funding Open Access funding enabled and organized by Projekt DEAL.

Open Access Dieser Artikel wird unter der Creative Commons Namensnennung 4.0 International Lizenz veröffentlicht, welche die Nutzung, Vervielfältigung, Bearbeitung, Verbreitung und Wiedergabe in jeglichem Medium und Format erlaubt, sofern Sie den/die ursprünglichen Autor(en) und die Quelle ordnungsgemäß nennen, einen Link zur Creative Commons Lizenz beifügen und angeben, ob Änderungen vorgenommen wurden.

Die in diesem Artikel enthaltenen Bilder und sonstiges Drittmaterial unterliegen ebenfalls der genannten Creative Commons Lizenz, sofern sich aus der Abbildungslegende nichts anderes ergibt. Sofern das betreffende Material nicht unter der genannten Creative Commons Lizenz steht und die betreffende Handlung nicht nach gesetzlichen Vorschriften erlaubt ist, ist für die oben aufgeführten Weiterverwendungen des Materials die Einwilligung des jeweiligen Rechteinhabers einzuholen.

Weitere Details zur Lizenz entnehmen Sie bitte der Lizenzinformation auf http://creativecommons.org/ licenses/by/4.0/deed.de. 


\section{Literatur}

Anders, Y., Kunter, M., Brunner, M., Krauss, S., \& Baumert, J. (2010). Diagnostische Fähigkeiten von Mathematiklehrkräften und ihre Auswirkungen auf die Leistungen ihrer Schülerinnen und Schüler [Mathematics teachers "diagnostic skills and their impact on students" achievements]. Psychologie in Erziehung und Unterricht, 57, 175-193.

Artelt, C., \& Gräsel, C. (2009). Diagnostische Kompetenz von Lehrkräften [Diagnostic competence of teachers]. Zeitschrift für Pädagogische Psychologie, 23(3), 157-160.

Artelt, C., \& Rausch, T. (2014). Accuracy of teacher judgements. When and for what reasons? In S. KrolakSchwerdt, S. Glock \& M. Böhmer (Hrsg.), Teachers' professional development: assessment, training, and learning (S. 27-43). Rotterdam: Sense Publishers.

Baumert, J., \& Kunter, M. (2013). The COACTIV model of teachers' professional compe-tence. In M. Kunter, J. Baumert, W. Blum, U. Klusmann, S. Krauss \& M. Neubrand (Hrsg.), Cognitive activation in the mathematics classroom and professional competence of teachers, mathematics teacher education (Bd. 8, S. 25-48). New York: Springer.

Black, P., \& Wiliam, D. (2003). 'In praise of educational research': formative assessment. British Educational Research Journal, 29(5), 623-637.

Brunswik, E. (1955). Representative design and probabilistic theory in a functional psychology. Psychological Review, 62, 193-217.

Feeney, A., \& Heit, E. (Hrsg.). (2007). Inductive reasoning: experimental, developmental, and computational approaches. New York: Cambridge University Press.

Gigerenzer, G., \& Gaissmaier, W. (2011). Heuristic decision making. Annual review of psychology, 62, 451-482.

Glock, S., Krolak-Schwerdt, S., \& Cate, P-t I. M. (2015). Are school placement recommendations accurate? The effect of students' ethnicity on teachers' judgments and recognition memory. European Journal of Psychology of Education, 30(2), 169-188.

Heitzmann, N., Seidel, T., Opitz, A., Hetmanek, A., Wecker, C., Fischer, M., et al. (2019). Facilitating diagnostic competences in simulations: A conceptual framework and a research agenda for medical and teacher education. Frontline Learning Research, 7(4), 1-24.

Helmke, A. (2010). Unterrichtsqualität und Lehrerprofessionalität. Diagnose, Evaluation und Verbesserung des Unterrichts [Teaching quality and teachers' professionalism. Diagnosis, evaluation and improvement of teaching]. Seelze: Klett.

Herppich, S., Praetorius, K., Förster, N., Glogger-Frey, I., Karst, K., Leutner, D., Behrmann, L., Böhmer, M., Ufer, S., Klug, J., Hetmanek, A., Ohle, A., Böhmer, I., Karing, C., Kaiser, J., \& Südkamp, A. (2018). Teachers' assessment competence: Integrating knowledge-, process-, and product-oriented approaches into a competence-oriented conceptual model. Teaching and Teacher Education, 76, 181-193.

Hobfoll, S.E., Halbesleben, J., Neveu, J.-P., \& Westman, M. (2018). Conservation of resources in the organizational context: the reality of resources and their consequences. Annual Review of Organizational Psychology and Organizational Behavior, 5, 103-128.

Hoge, R.D., \& Coladarci, T. (1989). Teacher-based judgements of academic achievement: a review of literature. Review of Educational Research, 59(3), 297-313.

Kahneman, D. (2003). A perspective on judgement and choice. American Psychologist, 58, 697-720.

Leuders, T., Dörfler, T., Leuders, J., \& Philipp, K. (2018). Diagnostic competence of mathematics teachers: unpacking a complex construct. In T. Leuders, T. Dörfler, J. Leuders \& K. Philipp (Hrsg.), Diagnostic competence of mathematics teachers. Unpacking a complex construct in teacher education and teacher practice (S. 3-32). New York: Springer.

Loibl, K., Leuders, T., \& Dörfler, T. (2020). A framework for explaining teachers' diagnostic Judgements by cognitive modeling (DiacoM). Teaching and Teacher Education. https://oi.org/10.1016/ j.tate.2020.103059.

Newell, A., \& Simon, H. A. (1972). Human problem solving. Englewood Cliffs: Prentice Hall.

Popham, W. J. (2009). Assessment literacy for teachers: faddish or fundamental? Theory into Practice, 48, $4-11$.

Praetorius, A. K., Berner, V.D., Zeinz, H., Scheunpflug, A., \& Dresel, M. (2013). Judgement confidence and judgement accuracy of teachers in judging self-concepts of students. Journal of Educational Research, 106, 64-76.

Schoenfeld, A.H. (1985). Mathematical problem-solving. New York: Academic Press. 
Schrader, F.-W. (1997). Lern- und Leistungsdiagnostik im Unterricht [Assessment in the classroom]. In F. E. Weinert (Hrsg.), Psychologie des Unterrichts und der Schule [Psychology of teaching and education] (S. 659-699). Göttingen: Hogrefe.

Schrader, F.-W. (2009). Anmerkungen zum Themenschwerpunkt Diagnostische Kompetenz von Lehrkräften. [The Diagnostic Competency of Teachers]. Zeitschrift für Pädagogische Psychologie, 23(34), 237-245.

Spinath, B. (2005). Akkuratheit der Einschätzung von Schülermerkmalen durch Lehrer und das Konstrukt der diagnostischen Kompetenz [Accuracy of teacher judgements on student characteristics and the construct of diagnostic competence]. Zeitschrift für Pädagogische Psychologie, 19, 85-95.

Südkamp, A., Kaiser, J., \& Möller, J. (2012). Accuracy of teachers' judgements of students' academic achievement: a meta-analysis. Journal of Educational Psychology, 104(3), 743-762.

Weinert, F.E., Schrader, F.-W., \& Helmke, A. (1990). Educational expertise. School Psychology International, 11, 163-180. 